\title{
Optimal Solution Approximation for Infinite Positive-Definite Quadratic Programming ${ }^{1}$
}

\author{
P. Benson, ${ }^{2}$ R. L. Smith, ${ }^{3}$ I. E. Schochetman, ${ }^{4}$ and J. C. Bean ${ }^{5}$ \\ Communicated by D. G. Luenberger
}

\begin{abstract}
We consider a general doubly-infinite, positive-definite, quadratic programming problem. We show that the sequence of unique optimal solutions to the natural finite-dimensional subproblems strongly converges to the unique optimal solution. This offers the opportunity to arbitrarily well approximate the infinite-dimensional optimal solution by numerically solving a sufficiently large finite-dimensional version of the problem. We then apply our results to a general time-varying, infinite-horizon, positive-definite, LQ control problem.
\end{abstract}

Key Words. Time-varying systems, positive-definite costs, infinitehorizon optimization, infinite quadratic programming, solution approximations, LQ control problems.

\section{Introduction}

Consider a general doubly-infinite quadratic programming problem of the following form:

$$
\begin{aligned}
\text { (P) } \min & \sum_{j=1}^{\infty}\left\langle x_{j}, Q_{j} x_{j}\right\rangle, \\
\text { s.t. } \quad & A_{i, i-1} x_{i-1}+A_{i i} x_{i}=b_{i}, \quad i=1,2, \ldots, \\
& x_{j} \in \mathfrak{R}^{n_{j}}, \quad j=1,2, \ldots,
\end{aligned}
$$

\footnotetext{
${ }^{\mathrm{I}}$ This work was supported in part by the National Science Foundation under Grants ECS8700836, DDM-9202849, and DDM-9214894.

${ }^{2}$ President, Rubicon, Ann Arbor, Michigan.

${ }^{3}$ Professor, Department of Industrial and Operations Engineering, University of Michigan, Ann Arbor, Michigan.

${ }^{4}$ Professor, Department of Mathematical Sciences, Oakland University, Rochester, Michigan. ${ }^{5}$ Professor, Department of Industrial and Operations Engineering, University of Michigan, Ann Arbor, Michigan.
} 
where $A_{10}$ is defined to be $0, Q_{j}$ is a symmetric matrix for each $j, b_{i} \in \Re^{m_{i}}$ for each $i$, and $A_{i, i-1}, A_{i i}$ are matrices of appropriate sizes for each $i$. The lowerstaircase structure of the constraint system is equivalent to the condition that each constraint contains finitely many variables and each variable appears in at most finitely many constraints. An important special case is the LQ regulator problem (Ref. 1).

As we shall see, we may, without loss of optimality, embed $(\mathrm{P})$ in the infinite-dimensional Hilbert space

$$
H=\left\{\left(x_{j}\right)_{j=1}^{\infty}: x_{j} \in \mathfrak{R}^{n_{j}}, j=1,2, \ldots ; \sum_{j=1}^{\infty}\left\|x_{j}\right\|_{2}^{2}<\infty\right\},
$$

the real Hilbert sum of the $\mathfrak{R}^{n_{j}}$. We assume that the linear operator $Q$, given by

$$
Q(x)=\left(Q_{j} x_{j}\right)_{j=1}^{\infty}, \quad x=\left(x_{j}\right)_{j=1}^{\infty} \in H,
$$

is a bounded operator mapping $H$ to itself. Note that $Q$ is a bounded operator if and only if

$$
\sup _{1 \leq j<\infty}\left\|Q_{j}\right\|_{2}<\infty
$$

where $\|\cdot\|_{2}$ is the matrix norm corresponding to the underlying Euclidean norm, also denoted by $\|\cdot\|_{2}$. Of course, $Q$ is also self-adjoint since each $Q_{j}$ is symmetric, $j=1,2, \ldots$.

Throughout this paper, we will assume that $Q$ is positive definite in the following sense. In conventional operator theory, an operator $T$ is positive definite if $\langle v, T(v)\rangle>0$, for all $v$ in the underlying Hilbert space $K$ different from zero. Here, $Q$ is positive definite in this sense if and only if $Q_{j}$ is a positive-definite matrix, for each $j=1,2, \ldots$ However, we adopt the stronger notion of positive definiteness offered in Refs. 2-3. See also Ref. 4, where the term positive bounded below is used. Thus, we will say that an operator $T$ is positive definite if there exists $\alpha_{T}>0$ such that

$$
\alpha_{T}\|v\|^{2} \leq\langle v, T(v)\rangle, \quad v \in K .
$$

This is a stronger notion, since for example, if $K$ is the space $l^{2}$ of squaresummable sequences and

$$
T(v)=\left((1 / i) v_{i}\right)_{i=1}^{\infty}, \quad v \in l^{2},
$$

then $T$ satisfies the former condition but not the latter. Moreover, it is not difficult to verify that, in general, $T$ is positive definite if and only if there 
exists $\alpha>0$ and a positive-semidefinite operator $R: K \rightarrow K$, i.e.,

$$
\langle v, R(v)\rangle \geq 0, \quad v \in K,
$$

such that

$$
\langle v, T(v)\rangle=\langle v, R(v)\rangle+\alpha\|v\|^{2}, \quad v \in K .
$$

In terms of the $Q_{j}$, it can be shown that our operator $Q$ is positive definite (in the stronger sense) if and only if each $Q_{j}$ is positive definite, for $j=$ $1,2, \ldots$, and the $\alpha_{Q_{j}}$ can be chosen so that

$$
\inf _{i \leq j<\infty} \alpha_{Q_{j}}>0 \text {. }
$$

We also assume that the feasible region $X$, where

$$
X=\left\{x \in H: A_{i, i-1} x_{i-1}+A_{i i} x_{i}=b_{i}, i=1,2, \ldots\right\},
$$

is nonempty. As we shall see in Section 2 , there is no loss of optimality in requiring that $X \subseteq H$, i.e., requiring that

$$
\sum_{j=1}^{\infty}\left\|x_{j}\right\|_{2}^{2}<\infty
$$

be included among the constraints. Of course, $X$ is closed and affine in $H$, and for each $x \in H$, we have

$$
\langle x, Q(x)\rangle=\sum_{j=1}^{\infty}\left\langle x_{j}, Q_{j} x_{j}\right\rangle
$$

For $Q$ positive definite, it is well known that $\langle\cdot, Q(\cdot)\rangle$ defines an inner product $\langle\cdot, \cdot\rangle_{Q}$ on $H$, with associated norm $\|\cdot\|_{Q}$ given by

$$
\|x\|_{Q}^{2}=\langle x, Q(x)\rangle, \quad x \in H .
$$

Since

$$
\alpha_{Q}\|x\|^{2} \leq\|x\|_{Q}^{2} \leq\|Q\|\|x\|^{2}, \quad x \in H,
$$

it follows that $\left(H,\langle\cdot,\rangle_{Q}\right)$ is a Hilbert space $H_{Q}$, which is the same set as $H$ and which is equivalent to $H$ as a normed space. Thus, (P) may be reformulated as follows:

(P) $\min _{x \in X}\|x\|_{Q}^{2}$.

Consequently, an optimal solution to $(\mathrm{P})$ is simply a best approximation in $X$ to the zero element of $H$, where $X$ is closed, convex, and nonempty. Such is well known to exist and be unique (Ref. 5). We let $x^{*}=\left(x_{j}^{*}\right)_{j=1}^{\infty}$ denote the unique solution to problem (P) in $H$. 
Our main objective in this paper is to approximate this optimal solution by the unique optimal solutions to the finite-dimensional subproblems $\left(\mathrm{P}_{\mathrm{N}}\right)$, $N=1,2, \ldots$, obtained by truncating (P) after $N$ vector variables and $N$ vector constraints. This is done in Section 2. In particular, we construct a sequence of optimal solutions to the subproblems $\left(\mathrm{P}_{\mathrm{N}}\right)$, which when embedded in $H$, converges to $x^{*}$, thus yielding solution convergence. Analogously, the corresponding sequence of optimal objective values converges to the objective value of $x^{*}$, thus yielding value convergence. These are the main results of this paper. In Section 3, we apply these results to a general time-varying, infinite-horizon, positive-definite, linear-quadratic control problem. This extends to the time-varying case the work of Lee, Chou, and Barr (Ref. 2), who demonstrated weak convergence of the finite-horizon optimal controls for the time-invariant case, and that of Zabczyk (Ref. 3), who later showed that this convergence is in fact strong. See also Schochetman and Smith (Ref. 6), who demonstrated strong convergence in the presence of a bounded control space.

\section{Approximation of Optimal Solutions}

Our objective in this section is to approximate the optimal solution $x^{*}$ of $(\mathrm{P})$ by optimal solutions to finite-dimensional subproblems of $(\mathrm{P})$. We will do the same for the corresponding objective function values.

We first observe however that there is no loss of optimality in requiring that the feasible region $X$ in problem (P) satisfy $X \subseteq H$, as opposed to $X \subseteq \Pi_{j=1}^{\infty} \Re^{n_{j}}$ more generally. The objective value $C(x)$ for $x \in \Pi_{j=1}^{\infty} \Re^{n_{j}}$ is given by

$$
C(x)=\sum_{j=1}^{\infty}\left\langle x_{j}, Q_{j} x_{j}\right\rangle
$$

where $0 \leq C(x) \leq \infty$. Since $Q$ is positive definite, there exists $\alpha_{Q_{j}}>0$, for each $j$, such that

$$
\begin{aligned}
& \alpha_{Q_{j}}\left\|x_{j}\right\|_{2}^{2} \leq\left\langle x_{j}, Q_{j} x_{j}\right\rangle, \\
& \inf _{1 \leq j<\infty} \alpha_{Q_{j}}>0 .
\end{aligned}
$$

Suppose that

$$
x \in \Pi_{j=1}^{\infty} \Re^{n_{j}} \text { and } x \notin H,
$$


i.e.,

$$
\sum_{j=1}^{\infty}\left\|x_{j}\right\|_{2}^{2}=\infty
$$

Then,

$$
\begin{aligned}
C(x) & =\sum_{j=1}^{\infty}\left\langle x_{j}, Q_{j} x_{j}\right\rangle \\
& \geq \sum_{j=1}^{\infty} \alpha_{Q_{j}}\left\|x_{j}\right\|_{2}^{2} \\
& \geq \inf _{1 \leq j<\infty} \alpha_{Q_{j}} \sum_{j=1}^{\infty}\left\|x_{j}\right\|_{2}^{2},
\end{aligned}
$$

which implies that $C(x)=\infty$ for $\operatorname{such} x$. Thus, if $Q$ is positive definite, there is no loss of optimality in requiring that the feasible solutions to $(\mathrm{P})$ be square summable. Moreover, since $X \neq \varnothing$, it follows that the optimal objective value $C^{*}=C\left(x^{*}\right)$ for $(\mathrm{P})$ satisfies $0 \leq C^{*}<\infty$, because

$$
C(x)=\langle x, Q(x)\rangle, \quad x \in H .
$$

Next, we construct finite-dimensional approximating subproblems of (P) corresponding to finite truncations of (P). Specifically, for each $N=$ $1,2, \ldots$, let $\left(P_{N}\right)$ be defined as follows:

$$
\begin{aligned}
\left(\mathbf{P}_{\mathrm{N}}\right) \quad \min & \sum_{j=1}^{N}\left\langle x_{j}, Q_{j} x_{j}\right\rangle, \\
\text { s.t. } & A_{i, i-1} x_{i-1}+A_{i i} x_{i}=b_{i}, \quad i=1,2, \ldots, N, \\
& x_{j} \in \mathfrak{R}^{n_{j}}, \quad j=1,2, \ldots, N .
\end{aligned}
$$

If we let $\Phi_{N}$ denote the feasible region to $\left(\mathrm{P}_{\mathrm{N}}\right)$, then $\Phi_{N}$ is a closed, affine subset of $\Pi_{j=1}^{N} \Re^{n_{j}}$, which is nonempty, since it must contain $\left(x_{1}^{*}, \ldots, x_{N}^{*}\right)$. Moreover, the operator $Q_{1} \times \cdots \times Q_{N}$ is positive definite on $\Pi_{j=1}^{N} \Re^{n_{j}}$. Consequently, as for $(\mathrm{P})$, there exists a unique solution in $\Phi_{N}$ to $\left(\mathrm{P}_{\mathrm{N}}\right)$, which we denote by $\left(\xi_{1}^{N}, \ldots, \xi_{N}^{N}\right)$. The optimal value $C_{N}^{*}$ for $\left(\mathrm{P}_{\mathrm{N}}\right)$ is then given by

$$
C_{N}^{*}=\sum_{j=1}^{N}\left\langle\xi_{j}^{N}, Q_{j} \xi_{j}^{N}\right\rangle, \quad N=1,2, \ldots
$$

Next, we embed $\left(\mathrm{P}_{\mathrm{N}}\right)$ in $H$. Let $X_{N}$ be the closed, affine space in $H$ given by

$$
X_{N}=\Phi_{N} \times\left(\oplus_{j=N+1}^{\infty} \Re^{n_{j}}\right), \quad N=1,2, \ldots ;
$$


i.e., $X_{N}$ consists of arbitrary square-summable extensions in $H$ of the elements of $\Phi_{N}$. Then,

$$
\begin{aligned}
& X_{N+1} \subseteq X_{N}, \quad N=1,2, \ldots, \\
& X=\bigcap_{N=1}^{\infty} X_{N} .
\end{aligned}
$$

In particular, $X_{N} \neq \varnothing$, all $N$.

Let $Q^{N}: H \rightarrow H$ be defined by

$$
Q^{N}(x)=\left(Q_{1} x_{1}, \ldots, Q_{N} x_{N}, 0, \ldots\right), \quad x \in H,
$$

so that $Q^{N}$ is a positive-semidefinite operator on $H$, for all $N$. Define $\left(\mathrm{P}^{\mathrm{N}}\right)$ to be the problem obtained by embedding $\left(\mathrm{P}_{\mathrm{N}}\right)$ in $H$, that is,

$$
\left(\mathrm{P}^{\mathrm{N}}\right) \min \quad C_{N}(x)=\left\langle x, Q^{N}(x)\right\rangle=\sum_{j=1}^{N}\left\langle x_{j}, Q_{j} x_{j}\right\rangle,
$$

s.t. $x \in X_{N}$.

Clearly, each $\left(\mathrm{P}^{\mathrm{N}}\right)$ has infinitely many optimal solutions $X_{N}^{*}$ in $X_{N}$ given by

$$
X_{N}^{*}=\left\{x \in H: x_{j}=\xi_{j}^{N}, j=1,2, \ldots, N ; x_{j} \text { arbitrary, } j>N\right\} .
$$

In particular, we let $\xi^{N}$ denote the element of $X_{N}^{*}$ given by

$$
\xi^{N}=\left(\xi_{1}^{N}, \ldots, \xi_{N}^{N}, 0,0, \ldots\right),
$$

so that $\xi^{N} \in H$, for all $N$, and

$$
C_{N}^{*}=\left\langle\xi^{N}, Q^{N}\left(\xi^{N}\right)\right\rangle, \quad N=1,2, \ldots
$$

It is important to note that although in general

$$
\left\langle x, Q^{N}(x)\right\rangle \leq\langle x, Q(x)\rangle=\|x\|_{Q}^{2}, \quad x \in H,
$$

since $\xi_{j}^{N}=0$, for $j>N$, we have that

$$
C_{N}^{*}=\left\langle\xi^{N}, Q^{N}\left(\xi^{N}\right)\right\rangle=\left\langle\xi^{N}, Q\left(\xi^{N}\right)\right\rangle=\left\|\xi^{N}\right\|_{Q}^{2}, \quad N=1,2, \ldots
$$

Lemma 2.1. The sequence $\left\{\xi^{N}\right\}_{N=1}^{\infty}$ is bounded in $H$.

Proof. Since $x^{*} \in X$, it follows that $x^{*} \in X_{N}$, for all $N$. Hence,

$$
C_{N}^{*} \leq\left\langle x^{*}, Q^{N}\left(x^{*}\right)\right\rangle, \quad N=1,2, \ldots
$$

But

$$
\begin{aligned}
\left\langle x^{*}, Q^{N}\left(x^{*}\right)\right\rangle & =\sum_{j=1}^{N}\left\langle x_{j}^{*}, Q_{j} x_{j}^{*}\right\rangle \\
& \leq \sum_{j=1}^{\infty}\left\langle x_{j}^{*}, Q_{j} x_{j}^{*}\right\rangle \\
& =\left\langle x^{*}, Q\left(x^{*}\right)\right\rangle, \quad N=1,2, \ldots,
\end{aligned}
$$

so that $\left\{\mathrm{C}_{N}^{*}\right\}_{N=1}^{\infty}$ is as bounded sequence of real numbers. 
Now, suppose that $\left\{\xi^{N}\right\}_{N=1}^{\infty}$ is not bounded in $H$, i.e., $\left\{\left\|\xi^{N}\right\|\right\}_{N=1}^{\infty}$ is not a bounded sequence of real numbers. Then, $\left\{\left\|\xi^{N}\right\|_{Q}\right\}_{N=1}^{\infty}$ is not bounded either. But

$$
\left\|\xi^{N}\right\|_{Q}^{2}=\left\langle\xi^{N}, Q\left(\xi^{N}\right)\right\rangle=\left\langle\xi^{N}, Q^{N}\left(\xi^{N}\right)\right\rangle=C_{N}^{*}, \quad N=1,2, \ldots,
$$

which is a contradiction.

The following is the main result of this section.

Theorem 2.1. If $Q$ is positive definite in problem (P), then the sequence $\left\{\xi^{N}\right\}_{N=1}^{\infty}$ converges strongly to $x^{*}$. In particular, the sequence $\left\{\xi_{j}^{N}\right\}_{N=1}^{\infty}$ converges to $x_{j}^{*}$, for each $j=1,2, \ldots$ Moreover, the sequence $\left\{C_{N}^{*}\right\}_{N=1}^{\infty}$ converges to $C^{*}$.

Proof. Let $\left\{\xi^{N_{k}}\right\}_{k=1}^{\infty}$ be an arbitrary subsequence of $\left\{\xi^{N}\right\}_{N=1}^{\infty}$, which is necessarily bounded also. By the Banach-Alaoglu theorem (Ref. 7), we may assume (passing to a subsequence if necessary) that $\left\{\xi^{N_{k}}\right\}_{k=1}^{\infty}$ converges weakly to some $y$ in $H$.

The constraints of $(\mathrm{P})$ have been given as a system of vector equations. However, each scalar constraint of $(\mathrm{P})$ is easily seen to be of the form $\langle a, x\rangle=b$, where $x \in H, b \in \mathfrak{R}$, and $a$ is of the form $\left(a_{1}, 0, \ldots\right)$, for $a_{1} \in \mathfrak{R}^{n_{1}}$, or of the form $\left(0, \ldots, 0, a_{i-1}, a_{i}, 0, \ldots\right)$, for $a_{i-1} \in R e^{n_{i-1}}$ and $a_{i} \in \mathfrak{R}^{n_{i}}$. Thus, $a$ is in $H$ in either case. Also, $\left\{\xi^{N_{k}}\right\}_{k=1}^{\infty}$ must eventually satisfy every such scalar constraint. Therefore, $\left\langle a, \xi^{N_{k}}\right\rangle=b$, for $k$ sufficiently large. However, $\left\langle a, \xi^{N_{k}}\right\rangle \rightarrow\langle a, y\rangle$, as $k \rightarrow \infty$, so that $\langle a, y\rangle=b$. Thus, $y$ must satisfy each constraint of $(\mathrm{P})$, i.e., $y \in X$ necessarily.

Next we show that $\left\{\xi^{N_{k}}\right\}_{k=1}^{\infty}$ converges strongly to $y$. By definition of the inner product on $H_{Q}$, it follows that $\left\{\xi^{N_{k}}\right\}_{k=1}^{\infty}$ also converges weakly to $y$ in $H_{Q}$. Since $y \in X$, it follows that $y \in X_{N_{k}}$, for all $k$, so that

$$
C_{N_{k}}^{*} \leq\left\langle y, Q^{N_{k}}(y)\right\rangle
$$

i.e.,

$$
\begin{aligned}
\left\|\xi^{N_{k}}\right\|_{Q}^{2} & =\left\langle\xi^{N_{k}}, Q\left(\xi^{N_{k}}\right)\right\rangle \\
& =\left\langle\xi^{N_{k}}, Q^{N_{k}}\left(\xi^{N_{k}}\right)\right\rangle \\
& =C_{N_{k}}^{*} \\
& \leq\left\langle y, Q^{N_{k}}(y)\right\rangle \\
& \leq\langle y, Q(y)\rangle \\
& =\|y\|_{Q}^{2}, \quad k=1,2, \ldots
\end{aligned}
$$


Thus,

$$
\left\|\xi^{N_{k}}\right\|_{Q} \leq\|y\|_{Q}, \quad k=1,2, \ldots
$$

Now, observe that a weakly convergent sequence in a Hilbert space is strongly convergent, provided the norms of the sequence elements are bounded by the norm of the weak limit (Ref. 8, p. 206). Consequently, $\left\{\xi^{N_{k}}\right\}_{k=1}^{\infty}$ converges strongly to $y$ in $H_{Q}$, and hence in $H$.

Next, we show that $y=x^{*}$. If not, then

$$
C\left(x^{*}\right)<C(y),
$$

i.e.,

$$
\left\|x^{*}\right\|_{Q}^{2}<\|y\|_{Q}^{2},
$$

because $y \in X$. Since $\left\{\xi^{N_{k}}\right\}_{N=1}^{\infty}$ converges strongly to $y$ in $H$, we have that

$$
\lim _{k \rightarrow \infty}\left\|\xi^{N_{k}}\right\|^{2}=\|y\|^{2},
$$

and hence,

$$
\lim _{k \rightarrow \infty}\left\|\xi^{N_{k}}\right\|_{Q}^{2}=\|y\|_{Q}^{2} .
$$

Thus, for large $k$,

$$
\left\|\xi^{N_{k}}\right\|_{Q}^{2}>\left\|x^{*}\right\|_{Q}^{2}
$$

But since $x^{*} \in X_{N_{k}}$, we have

$$
C_{N_{k}}\left(\xi^{N_{k}}\right) \leq C_{N_{k}}\left(x^{*}\right),
$$

for all $k$. Therefore,

$$
\begin{aligned}
\left\|\xi^{N_{k}}\right\|_{Q}^{2} & =\left\langle\xi^{N_{k}}, Q\left(\xi^{N_{k}}\right)\right\rangle \\
& =\left\langle\xi^{N_{k}}, Q^{N_{k}}\left(\xi^{N_{k}}\right)\right\rangle \\
& =C_{N_{k}}\left(\xi^{N_{k}}\right) \\
& \leq C_{N_{k}}\left(x^{*}\right) \\
& =\left\langle x^{*}, Q^{N_{k}}\left(x^{*}\right)\right\rangle \\
& \leq\left\langle x^{*}, Q\left(x^{*}\right)\right\rangle \\
& =\left\|x^{*}\right\|_{Q}^{2}, \quad k=1,2, \ldots,
\end{aligned}
$$


so that

$$
\left\|\xi^{N_{k}}\right\|_{Q}^{2} \leq\left\|x^{*}\right\|_{Q}^{2}, \quad \text { all } k
$$

a contradiction. Hence, $y=x^{*}$ and $\xi^{N_{k}} \rightarrow x^{*}$ in $H$, as $k \rightarrow \infty$.

We have shown that an arbitrary subsequence of $\left\{\xi^{N}\right\}_{N=1}^{\infty}$ has a subsequence which converges to $x^{*}$ in $H$. Thus, $\left\{\xi^{N}\right\}_{N=1}^{\infty}$ converges to $x^{*}$ in $H$. Consequently,

$$
\lim _{N \rightarrow \infty} \xi_{j}^{N}=x_{j}^{*}, \quad j=1,2, \ldots
$$

Finally, since $\xi^{N} \rightarrow x^{*}$ in $H_{Q}$ necessarily, we have that

$$
\begin{aligned}
C_{N}^{*} & =\left\langle\xi^{N}, Q^{N}\left(\xi^{N}\right)\right\rangle \\
& =\left\langle\xi^{N}, Q\left(\xi^{N}\right)\right\rangle \\
& =\left\|\xi^{N}\right\|_{Q}^{2}
\end{aligned}
$$

converges to $\left\|x^{*}\right\|_{Q}^{2}=C^{*}$, as $N \rightarrow \infty$.

To summarize the main result of this section for problem $(P)$, in the case of $Q$ positive definite, $(\mathrm{P})$ admits a unique solution $x^{*}$ which may be arbitrarily well approximated by solving sufficiently large finite-dimensional versions of $(\mathrm{P})$.

\section{Application to Control Theory}

Consider the following time-varying, infinite-horizon, linear quadratic control problem:

(C) $\min \sum_{j=1}^{\infty}\left[y_{j}^{t} S_{j} y_{j}+u_{j-1}^{t} R_{j-1} u_{j-1}\right]$,

$$
\begin{array}{ll}
\text { s.t. } & y_{i}=A_{i-1} y_{i-1}+B_{i-1} u_{i-1}+d_{i}, \quad i=1,2, \ldots, \\
& y_{j} \in \mathfrak{R}^{n}, \quad u_{j-1} \in \mathfrak{R}^{m}, \quad j=1,2, \ldots,
\end{array}
$$

where $y_{0}$ is given and each $d_{i}$ is a known exogenous parameter (e.g., demand in production-inventory models). For the $j$ th period, $y_{j}$ is the $j$ th state and $u_{j}$ is the resulting $j$ th control. Note that the cost of the initial state $y_{0}$ is constant and may be omitted. This problem is essentially an infinite-horizon version of the familiar LQ regulator problem (Ref. 1). 
Problem (C) may, without loss of optimality, be rewritten as follows:

(C) $\min \sum_{j=1}^{\infty}\left\langle\left[\begin{array}{l}y_{j} \\ u_{j-1}\end{array}\right],\left[\begin{array}{ll}S_{j} & 0 \\ 0 & R_{j-1}\end{array}\right]\left[\begin{array}{l}y_{j} \\ u_{j-1}\end{array}\right]\right\rangle$

$$
\begin{aligned}
& \text { s.t. }\left[-I, B_{0}\right]\left[\begin{array}{l}
y_{1} \\
u_{0}
\end{array}\right]=A_{0} y_{0}-d_{1}, \quad i=1 \text {, } \\
& {\left[A_{i-1}, 0\right]\left[\begin{array}{l}
y_{i-1} \\
u_{i-2}
\end{array}\right]+\left[-I, B_{i-1}\right]\left[\begin{array}{l}
y_{i} \\
u_{i-1}
\end{array}\right]} \\
& =-d_{i}, \quad i=2,3, \ldots, \\
& {\left[\begin{array}{l}
y_{j} \\
u_{j-1}
\end{array}\right] \in \mathfrak{R}^{n+m}, \quad j=1,2, \ldots,}
\end{aligned}
$$

where, in this case,

$$
\begin{aligned}
& x_{j}=\left[\begin{array}{l}
y_{j} \\
u_{j-1}
\end{array}\right], \quad j=1,2, \ldots, \\
& Q_{j}=\left[\begin{array}{ll}
S_{j} & 0 \\
0 & R_{j-1}
\end{array}\right], \quad j=1,2, \ldots, \\
& A_{i, i-1}=\left[A_{i-1}, 0\right], \quad i=2,3, \ldots, \\
& A_{i i}=\left[-I, B_{i-1}\right], \quad i=1,2, \ldots \text {, } \\
& b_{1}=A_{0} y_{0}-d_{1}, \quad i=1 \text {, } \\
& b_{i}=-d_{i}, \quad i=2,3, \ldots \text {, } \\
& H=\left\{\left(\left[\begin{array}{l}
y_{j} \\
u_{j-1}
\end{array}\right]\right)_{j=1}^{\infty}:\left[\begin{array}{l}
y_{j} \\
u_{j-1}
\end{array}\right] \in \mathfrak{R}^{n+m}\right. \\
& \left.j=1,2, \ldots ; \sum_{j=1}^{\infty}\left[\left\|y_{j}\right\|_{2}^{2}+\left\|u_{j-1}\right\|_{2}^{2}\right]<\infty\right\}, \\
& Q\left(\left(\left[\begin{array}{l}
y_{j} \\
u_{j-1}
\end{array}\right]\right)_{j=1}^{\infty}\right)=\left(\left[\begin{array}{ll}
S_{j} & 0 \\
0 & R_{j-1}
\end{array}\right]\left[\begin{array}{l}
y_{j} \\
u_{j-1}
\end{array}\right]\right)_{j=1}^{\infty},\left(\left[\begin{array}{l}
y_{j} \\
u_{j-1}
\end{array}\right]\right)_{j=1}^{\infty} \in H .
\end{aligned}
$$




\section{Lemma 3.1. If}

$$
\sup _{1 \leq j<\infty}\left\|S_{j}\right\|_{2}<\infty \text { and } \sup _{1 \leq j<\infty}\left\|R_{j-1}\right\|_{2}<\infty,
$$

then $Q$ is a bounded linear operator on $H$.

Proof. In this case, $\sup _{1 \leq j<\infty}\left\|Q_{j}\right\|_{2}$ is bounded by the maximum of $\sup _{1 \leq j<\infty}\left\|S_{j}\right\|_{2}$ and $\sup _{1 \leq j<\infty}\left\|R_{j-1}\right\|_{2}$.

Lemma 3.2. If the $S_{j}$ and $R_{j-1}, j=1,2, \ldots$, are positive definite and the $\alpha_{S_{j}}$ and $\alpha_{R_{j-1}}$ can be chosen such that

$$
\inf _{1 \leq j<\infty} \alpha_{S_{j}}>0 \text { and } \inf _{1 \leq j<\infty} \alpha_{R_{j-1}}>0,
$$

then $Q$ is positive definite on $H$.

Proof. In this case, each $Q_{j}$ is positive definite and $\inf _{1 \leq j<\infty} \alpha_{Q_{j}}$ is bounded below by the minimum of $\inf _{1 \leq j<\infty} \alpha_{S_{j}}$ and $\inf _{1 \leq j<\infty} \alpha_{R_{j-1}}$.

We assume that the matrices $S_{j}$ and $R_{j-1}$ are symmetric, so that each matrix $Q_{j}$ is symmetric, $j=1,2, \ldots$ We also assume the hypotheses of Lemmas 3.1 and 3.2, so that $Q$ is a bounded linear operator on $H$ which is positive definite. Finally, we assume that the feasible region $X$ (contained in $H$ ) is nonempty.

As in Section 2, problem (C) has a unique solution $x^{*}=\left(x_{j}^{*}\right)_{j=1}^{\infty}$ in $X$ for

$$
x_{j}^{*}=\left[\begin{array}{l}
y_{j}^{*} \\
u_{j-1}^{*}
\end{array}\right], \quad j=1,2, \ldots,
$$

where for each stage $j, y_{j}^{*} \in \mathfrak{R}^{n}$ is the resulting optimal state and $u_{j-1}^{*} \in \mathfrak{R}^{m}$ is the optimal control. For convenience, we let

$$
y^{*}=\left(y_{j}^{*}\right)_{j=1}^{\infty} \text { and } u^{*}=\left(u_{j-1}^{*}\right)_{j=1}^{\infty} .
$$

Our objective here is to approximate the vector $y^{*}$ of optimal states and the vector $u^{*}$ of optimal controls by corresponding optimal solutions for finite truncations of (C). We will also approximate the optimal cost,

$$
C^{*}=\sum_{j=1}^{\infty}\left[\left(y_{j}^{*}\right)^{t} S_{j} y_{j}^{*}+\left(u_{j-1}^{*}\right)^{t} R_{j-1} u_{j-1}^{*}\right]
$$

for (C) by the optimal costs to these finite truncations. 
To this end, for each $N=1,2, \ldots$, let $\left(\mathrm{C}_{\mathrm{N}}\right)$ denote the finite-dimensional truncation of $(C)$ which is analogous to $\left(P_{N}\right)$, i.e.,

$\left(\mathrm{C}_{\mathrm{N}}\right) \min \sum_{j=1}^{N}\left[y_{j}^{t} S_{j} y_{j}+u_{j-1}^{t} R_{j-1} u_{j-1}\right]$,

s.t. $\quad y_{i}=A_{i-1} y_{i-1}+B_{i-1} u_{i-1}+d_{i}, \quad i=1,2, \ldots, N$,

$y_{j} \in \mathfrak{R}^{n}, \quad u_{j-1} \in \mathfrak{R}^{m}, \quad j=1,2, \ldots, N$,

with $y_{0}$ given. As above, $\left(\mathrm{C}_{\mathrm{N}}\right)$ can be rewritten as

$\left(\mathrm{C}_{\mathrm{N}}\right) \min \sum_{j=1}^{N}\left\langle\left[\begin{array}{l}y_{j} \\ u_{j-1}\end{array}\right],\left[\begin{array}{ll}S_{j} & 0 \\ 0 & R_{j-1}\end{array}\right]\left[\begin{array}{l}y_{j} \\ u_{j-1}\end{array}\right]\right\rangle$,

s.t. $\left[-I, B_{0}\right]\left[\begin{array}{l}y_{1} \\ u_{0}\end{array}\right]=A_{0} y_{0}-d_{1}, \quad i=1$,

$$
\begin{aligned}
& {\left[A_{i-1} 0\right]\left[\begin{array}{l}
y_{i-1} \\
u_{i-2}
\end{array}\right]+\left[-I, B_{i-1}\right]\left[\begin{array}{l}
y_{i} \\
u_{i-1}
\end{array}\right]=-d_{i}, \quad i=2,3, \ldots, N,} \\
& {\left[\begin{array}{l}
y_{j} \\
u_{j-1}
\end{array}\right] \in \mathfrak{R}^{n+m}, \quad j=1,2, \ldots, N \text {. }}
\end{aligned}
$$

As in Section 2, for each $N$, problem $\left(\mathrm{C}_{\mathrm{N}}\right)$ has a unique solution $\left(\xi_{1}^{N}, \ldots, \xi_{N}^{N}\right)$, where this time

$$
\xi_{j}^{N}=\left[\begin{array}{l}
\eta_{j}^{N} \\
\mu_{j-1}^{N}
\end{array}\right]
$$

for $\eta_{j}^{N} \in \mathfrak{R}^{n}$, the optimal state for $\left(\mathrm{C}_{\mathrm{N}}\right)$ beginning stage $j$, and $\mu_{j-1}^{N} \in \mathfrak{R}^{m}$, the optimal control for $\left(\mathrm{C}_{\mathrm{N}}\right)$ at stage $j$, for $j=1,2, \ldots, N$. As in Section 2, we define

$$
\xi^{N}=\left(\xi_{1}^{N}, \ldots, \xi_{N}^{N}, 0, \ldots\right), \quad N=1,2, \ldots
$$

Analogously, we let

$$
\begin{array}{ll}
\eta^{N}=\left(\eta_{1}^{N}, \ldots, \eta_{N}^{N}, 0, \ldots\right), & N=1,2, \ldots, \\
\mu^{N}=\left(\mu_{1}^{N}, \ldots, \mu_{N}^{N}, 0, \ldots\right), & N=1,2, \ldots
\end{array}
$$


The corresponding optimal cost $C_{N}^{*}$ for $\left(\mathrm{C}_{\mathrm{N}}\right)$ is then

$$
\sum_{j=1}^{N}\left[\left(\eta_{j}^{N}\right)^{t} S_{j} \eta_{j}^{N}+\left(\mu_{j-1}^{N}\right)^{t} R_{j-1} \mu_{j-1}^{N}\right], \quad N=1,2, \ldots
$$

We next apply the main results of Section 2 to problem (C).

Theorem 3.1. Suppose that, for problem (C), the matrices $S_{j}$ and $R_{j-1}$ are symmetric and positive definite, for each $j=1,2, \ldots$, and satisfy the hypotheses of Lemmas 3.1 and 3.2. Suppose that the feasible region for problem (C) is nonempty. Then, the state sequence $\left\{\eta^{N}\right\}_{N=1}^{\infty}$ converges strongly to the vector $y^{*}$ of optimal states, i.e.,

$$
\sum_{j=1}^{\infty}\left\|\eta_{j}^{N}-y_{j}^{*}\right\|_{2}^{2} \rightarrow 0, \quad N \rightarrow \infty
$$

and the control sequence $\left\{\mu^{N}\right\}_{N=1}^{\infty}$ converges strongly to the vector $\mu^{*}$ of optimal controls, i.e.,

$$
\sum_{j=1}^{\infty}\left\|\mu_{j-1}^{N}-\mu_{j-1}^{*}\right\|_{2}^{2} \rightarrow 0, \quad N \rightarrow \infty .
$$

In particular, for each $j=1,2, \ldots$, the sequence $\left\{\eta_{j}^{N}\right\}_{N=1}^{\infty}$ converges to $y_{j}^{*}$ and the sequence $\left\{\mu_{j-1}^{N}\right\}_{N=1}^{\infty}$ converges $\mu_{j-1}^{*}$. Finally, the cost sequence $\left\{C_{N}^{*}\right\}$ converges to the optimal cost $C^{*}$, i.e.,

$$
\begin{aligned}
& \sum_{j=1}^{N}\left[\left(\eta_{j}^{N}\right)^{t} S_{j} \eta_{j}^{N}+\left(\mu_{j-1}^{N}\right)^{t} R_{j-1} \mu_{j-1}^{N}\right] \\
& \rightarrow \sum_{j=1}^{\infty}\left[\left(y_{j}^{*}\right)^{t} S_{j} y_{j}^{*}+\left(u_{j-1}^{*}\right)^{t} R_{j-1} \mu_{j-1}^{*}\right], \quad \text { as } N \rightarrow \infty .
\end{aligned}
$$

Proof. Apply Theorem 2.2.

\section{References}

1. Lewis, F. L., Optimal Control, Wiley, New York, New York, 1986.

2. LeE, K. Y., Chow, S., and BARR, R. O., On the Control of Discrete-Time Distributed Parameter Systems, SIAM Journal on Control, Vol. 10, pp. 361-376, 1972.

3. ZАBCZYK, J., Remarks on the Control of Discrete-Time Distributed Parameter Systems, SIAM Journal on Control, Vol. 12, pp. 721-773, 1974.

4. Milne, R. D., Applied Functional Analysis, Pitman, Boston, Massachusetts, 1980.

5. Aubin, J. P., Applied Functional Analysis, Wiley, New York, New York, 1979. 
6. Schochetman, I. E., and Smith, R. L., Solution Approximation in InfiniteHorizon Linear Quadratic Control, IEEE Transactions on Automatic Control, Vol. 39, pp. 596-601, 1994.

7. Dunford, N., and Schwartz, J. T., Linear Operators, Vol. 1, Wiley-Interscience, New York, New York, 1964.

8. Kolmogorov, A. N., and Fomin, S. V., Introductory Real Analysis, PrenticeHall, Englewood Cliffs, New Jersey, 1970. 